
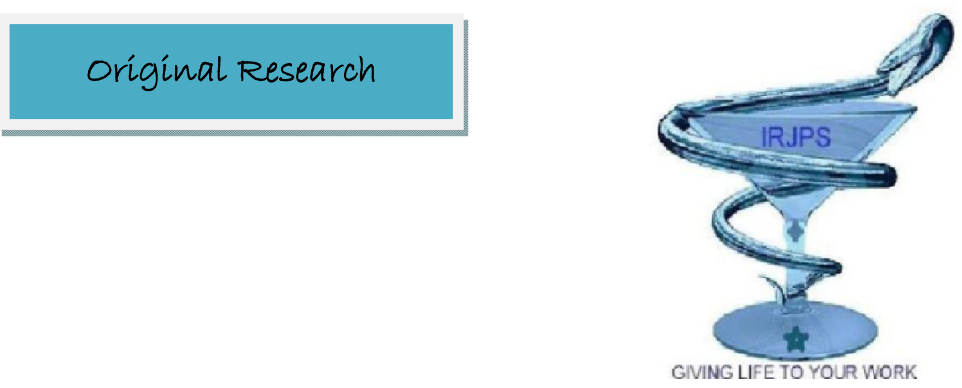

\title{
Evaluation Of Antimicrobial Activity Of Novel Series Of Ortho Hydroxy Chalcones
}

\author{
Bushra Ahmed Kateb ${ }^{1-2^{*}}$, Abdulkareem Ali Hussien ${ }^{1-2}$, M. A. Baseer ${ }^{1}$ \\ ${ }^{1}$ P. G. Research Center, Department of Chemistry, Yeshwant Mahavidyalaya , Nanded-431602 \\ ${ }^{2}$ Hodiedah University, Education College, Yemen.
}

\begin{abstract}
Numbers of Novel Chalcones were synthesized by reacting several substituted aryl aldehydes and ortho hydroxyl acetophenone, prepared by Claisen-Schmidt condensation reaction in $\mathrm{NaOH}$ solution in ethanol. The synthesized Chalcones compounds were characterized by Physical and spectral methods IR, ${ }^{1} \mathrm{H}-\mathrm{NMR}$ and Mass analysis. All the synthesized compounds have been screened and evaluated for antibacterial activity against Staphylococcus aureus $\mathrm{gr}+\mathrm{ve}$, Escherichia coli gr -ve Bacillus subtilis gr +ve, Salmonella typhi $\mathrm{gr}-\mathrm{ve}$, and antifungal activity against Aspergillus oryzoe, Aspergillus niger, using disc diffusion method. Most of the compounds showed significant antibacterial and antifungal activities. In this article efforts have been made to throw some light on the synthesis and biological activities of chalcones.
\end{abstract}

Keywords: Ortho hdroxy Chalcones, Synthesis, Antimcrobial Activity.

Corresponding Author: Bushra Ahmed Kateb

E-mail: nice1422@gmail.com
Indian Research Journal of Pharmacy and Science; 11(2016) 868-874 Journal Home Page: https://www.irjps.in 


\section{INTRODUCION}

The Chemistry of chalcones has generated intensive scientific studies throughout the world. Especially interest has been focused on the synthesis and biodynamic activities of chalcones. Novel chalcones were synthesized because it is known to exhibit various biological activities. Chalcones basic structure includes two aromatic ring bound by an $\alpha$, $\beta$-unsaturated carbonyl group, a unique template associated with very diverse application ${ }^{1}$. Chalcones (trans-1, 3-diaryl-2propen-1-ones) are $\alpha, \beta$ unsaturated ketones consisting of two aromatic rings (ring A and B) having diverse array of substituents. Rings are interconnected by a highly electrophonic three carbon $\alpha$, $\beta$-unsaturated carbonyl system that assumes linear or nearly planar structure ${ }^{2-4}$. They contain the ketoethylenic group $(-\mathrm{CO}-\mathrm{CH}=\mathrm{CH}-)$. Chalcones possess conjugated double bonds and a completely delocalized $\pi$-electron system on both benzene rings. Chalcones have been used as intermediate for the preparations of compounds having therapeutic value ${ }^{5-7}$. Due to the presence of enone functionality in chalcone moiety confers biological activity upon it, like anti-inflammatory ${ }^{8}$, antifungal 9 , antioxidant 10 , antimalarial ${ }^{11}$, antituberculosis ${ }^{12}$, analgesic ${ }^{13}$, anti HIV ${ }^{14}$ and antitumor ${ }^{15}$ activities. Different methods are available for the preparation of chalcones ${ }^{16-18}$. The most convenient method is the Claisen-Schimdt condensation of equimolar quantities of arylmethylketone with aryl aldehyde in the presence of alcoholic alkali ${ }^{19}$.

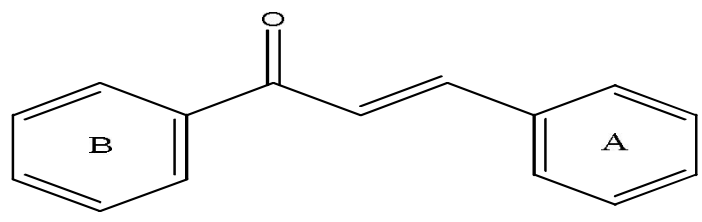

Figure-1: Chalcone

\section{MATERIALS AND METHODS:}

\section{Claisen-Schmidt condensation}

The most convenient method is the Claisen Schimdt condensation of equimolar quantities of aryl ketone with aryl aldehyde in the presence of alcoholic alkali $^{19}$.

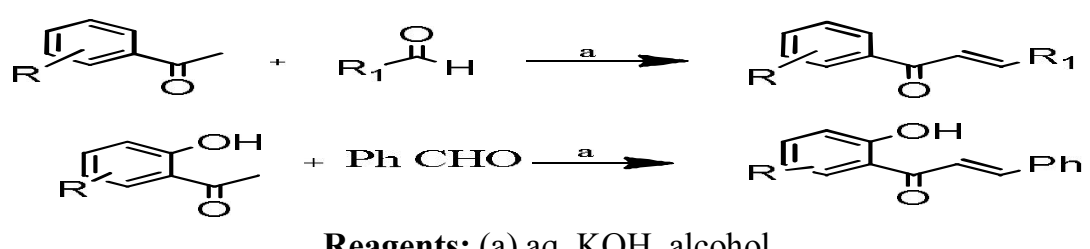

Reagents: (a) aq. $\mathrm{KOH}$, alcohol

\section{Experimental}

Melting points of the compounds were determined in open capillary tubes and are uncorrected, IR Spectra were recorded on Shimadzu FT-IR Spectrometer using potassium bromide pellets, ${ }^{1} \mathrm{H}$ NMR was determined on a Bruker Avance II 400 Spectrometer against TMS as internal standard. Mass spectra were recorded on waters Micromass Q-T of Micro spectrometry.

\section{General method for the synthesis of chalcones}

A mixture of Ortho hdroxy acetophenone $(1 \mathrm{mmol})$, substituted aryl aldehyde $(1 \mathrm{mmol})$ and $\mathrm{KOH}(2$ mmol, in minium $\mathrm{H}_{2} \mathrm{O}$ ) were taken in ethanol and stirred for one hour in cool condition. The completion of reaction was monitored by TLC. The products were isolated by acidification of the cool diluted acid solution and obtained solid product was filtered and washed with water and recrystallize by ethanol to get pure product. 
Scheme-1<smiles>[R]c1c([R2])c([R])c(C(C)=O)c([R])c1[R]</smiles>

2<smiles>[R]c1c([R])c([R])c(C(=O)C=[V])c(/C=C/[Al])c1[R]</smiles>

Chalcone $(3 a-j)$

$$
\mathbf{R}_{1}=\mathrm{OH}, \mathbf{R}_{2}=\mathrm{I}, \mathbf{R}_{3}=\mathbf{H}, \mathbf{R}_{\mathbf{4}}=\mathrm{Br}
$$

\section{RESULT AND DISCUSSION:}

The newly chalcones were carried out according to the Claisen-Schmidt condensation of ortho hydroxyl ketones with several aromatic aldehyde as indicated to Scheme1. The corresponding reactions proceeded smoothly and in good to excellent yields (65-90\%). The newly synthesized chalcones were characterized by their chemical, physical and spectral analysis data and are further subjected to antimicrobial studies which exhibit moderate to good activity.

Table1. Physical data of synthesized Chalcones

\begin{tabular}{ccccc}
\hline Comp.no & Product & Mol. Formula & Yield \% & M.P. $\left({ }^{\circ} \mathbf{C}\right)$ \\
$\mathbf{1}$ & $4 \mathrm{a}$ & $\mathrm{C}_{15} \mathrm{H}_{9} \mathrm{IO}_{2} \mathrm{ClBr}$ & 90 & 178 \\
$\mathbf{2}$ & $4 \mathrm{~b}$ & $\mathrm{C}_{15} \mathrm{H}_{10} \mathrm{O}_{3} \mathrm{IBr}$ & 80 & 158 \\
$\mathbf{3}$ & $4 \mathrm{c}$ & $\mathrm{C}_{15} \mathrm{H}_{9} \mathrm{O}_{2} \mathrm{IBr}_{2}$ & 70 & 198 \\
$\mathbf{4}$ & $4 \mathrm{~d}$ & $\mathrm{C}_{15} \mathrm{H}_{9} \mathrm{O}_{4} \mathrm{INBr}$ & 75 & 210 \\
$\mathbf{5}$ & $4 \mathrm{e}$ & $\mathrm{C}_{15} \mathrm{H}_{9} \mathrm{O}_{5} \mathrm{INBr}$ & 75 & 185 \\
$\mathbf{6}$ & $4 \mathrm{f}$ & $\mathrm{C}_{14} \mathrm{H}_{10} \mathrm{BrlSO}$ & 80 & 122 \\
$\mathbf{7}$ & $4 \mathrm{~g}$ & $\mathrm{C}_{13} \mathrm{H}_{9} \mathrm{O}_{2} \mathrm{INBr}$ & 80 & 120 \\
$\mathbf{8}$ & $4 \mathrm{~h}$ & $\mathrm{C}_{17} \mathrm{H}_{11} \mathrm{O}_{2} \mathrm{IBrN}$ & 70 & 130 \\
$\mathbf{9}$ & $4 \mathrm{i}$ & $\mathrm{C}_{15} \mathrm{H}_{9} \mathrm{IO}_{2} \mathrm{ClBr}$ & 70 & 112 \\
$\mathbf{1 0}$ & $4 \mathrm{j}$ & $\mathrm{C}_{15} \mathrm{H}_{9} \mathrm{O}_{2} \mathrm{IBr}_{2}$ & 65 & 128 \\
\hline
\end{tabular}


Scheme-1. Synthesis of Chalcones<smiles>O=C(/C=C/c1ccc(Cl)cc1)c1cc(Br)cc(I)c1O</smiles>

Figure-2 E)-1-(5-bromo-2-hydroxy-3-iodophenyl)-3-(4chlorophenyl)prop-2-en-1-one<smiles>O=C(/C=C/c1ccc(Br)cc1)c1cc(Br)cc(I)c1O</smiles>

Figure-4 (E)-3-(4-bromophenyl)-1-(5-bromo-2hydroxy-3-iodophenyl)prop-2-en-1-one<smiles>O=C(/C=C/c1ccc([N+](=O)[O-])c(O)c1)c1cc(Br)cc(I)c1O</smiles>

Figure-6 (E)-1-(5-bromo-2-hydroxy-3-iodophenyl)-3-(3-hydroxy-4nitrophenyl)prop-2-en-1-one<smiles>O=C(/C=C/c1cccnc1)c1cc(Br)cc(I)c1O</smiles>

Figure-8 (E)-1-(5-bromo-2-hydroxy-3-iodophenyl)-3-(pyridin-3-yl) prop-2-en-1-one<smiles>O=C(/C=C/c1ccccc1Cl)c1cc(Br)cc(I)c1O</smiles>

Figure-10 (E)-1-(5-bromo-2-hydroxy-3-iodophenyl)-3-(2chlorophenyl)prop-2-en-1-one<smiles>O=C(/C=C/c1ccc(O)cc1)c1cc(Br)cc(I)c1O</smiles>

Figure-3 (E)-1-(5-bromo-2-hydroxy-3-iodophenyl)-3-(4hydroxyphenyl)prop-2-en-1-one<smiles>O=C(/C=C/c1ccc([N+](=O)[O-])cc1)c1cc(Br)cc(I)c1O</smiles>

Figure-5 (E)-1-(5-bromo-2-hydroxy-3-iodophenyl)-3-(4 nitrophenyl)prop-2-en-1-one<smiles>Cc1ccc(/C=C/C(=O)c2cc(Br)cc(I)c2O)s1</smiles>

Figure-7 (E)-1-(5-bromo-2-hydroxy-3-iodophenyl)-3-(5methylthiophen-2-yl)prop-2-en-1-one<smiles>O=C(/C=C/c1c[nH]c2ccccc12)c1cc(Br)cc(I)c1O</smiles>

Figure-9 (E)-1-(5-bromo-2-hydroxy-3-iodophenyl)-3-(1 $H$-indol-3yl)prop-2-en-1-one<smiles>O=C(/C=C/c1ccccc1Br)c1cc(Br)cc(I)c1O</smiles>

Figure-11 ( $E$ )-1-(5-bromo-2-hydroxy-3-iodophenyl)-3-(2bromophenyl)prop-2-en-1-one 


\section{Spectral analysis of the compounds:}

The structures of some the compounds were established from IR, ${ }^{1} \mathrm{HNMR}$ and mass analysis.<smiles>Cc1c(Br)ccc(Cl)c1C(=O)C(Br)=Cc1c(Cl)ccc(Br)c1O</smiles>

$3 a$

Compound 3a :-

FTIR (KBr, $\left.\mathbf{~ c m}^{-1}\right): \quad 1634(\mathrm{C}=\mathrm{O}), 1562(\mathrm{C}=\mathrm{C})$, 1428(C-C Aromatic str), 815(C-Cl).

M.S. (m/z): 462(M-1), 464(M+1).

${ }^{1}$ HNMR:- $6.95\left(\mathrm{~d}, 1 \mathrm{H}, \mathrm{H}_{1}\right), 7.45\left(\mathrm{~s}, 1 \mathrm{H}, \mathrm{H}_{2}\right), 7.63(\mathrm{~d}$, $\left.1 \mathrm{H}, \mathrm{H}_{4}\right), 7.66\left(\mathrm{~d}, 1 \mathrm{H}, \mathrm{H}_{3}\right), 7.79(\mathrm{~d}, 1 \mathrm{H}, \mathrm{H \alpha}, \mathrm{J}=15 \mathrm{~Hz})$, 7.92(d, $\left.1 \mathrm{H}, \mathrm{H}_{5}\right), 8.12\left(\mathrm{~s}, 1 \mathrm{H}, \mathrm{H}_{6}\right)$ 8.14(d, $1 \mathrm{H}, \mathrm{H}$ $\beta, \mathrm{J}=15 \mathrm{~Hz}), 12.35(\mathrm{~s}, 1 \mathrm{H}, \mathrm{OH}$ ortho).<smiles>Cc1ccccc1/C(Cl)=C(\Br)C(=O)c1c(C)c(Br)c(C)c([N+](=O)[O-])c1O</smiles>

\section{Compound 3d :-}

FTIR (KBr, $\left.\quad \mathbf{c m}^{-1}\right): \quad 1614(\mathrm{C}=\mathrm{O}) \quad, 1573(\mathrm{C}=\mathrm{C})$, 1429(C-C Aromatic str) ,1307( $\left.\mathrm{NO}_{2}\right), 668(\mathrm{C}-\mathrm{Br})$.

M.S. (m/z): 490( $\left.\mathrm{M}^{+}\right)$, 489(M-1).

${ }^{1}$ HNMR:- 6.96(d, $\left.1 \mathrm{H}, \mathrm{H}_{1}\right), 7.61\left(\mathrm{~s}, 1 \mathrm{H}, \mathrm{H}_{3}\right) 7.63(\mathrm{~s}, 1 \mathrm{H}$, $\left.\mathrm{H}_{2}\right), \quad 7.71(\mathrm{~d}, \quad 1 \mathrm{H}, \quad \mathrm{H \alpha}, \mathrm{J}=15 \mathrm{~Hz}), \quad 8.02(\mathrm{~d}, \quad 1 \mathrm{H}, \quad \mathrm{H}$ $\beta, \mathrm{J}=15 \mathrm{~Hz}) 8.16\left(\mathrm{~s}, 1 \mathrm{H}, \mathrm{H}_{4}\right), 8.31\left(\mathrm{~s}, 1 \mathrm{H}, \mathrm{H}_{5}\right), 8.59(\mathrm{~s}, 1 \mathrm{H}$, $\mathrm{OH}), 12.26(\mathrm{~s}, 1 \mathrm{H}, \mathrm{OH}$ ortho).<smiles>Cc1ccc(/C(Cl)=C(\Br)C(=O)c2c(C)c(Br)c(C)c(I)c2O)s1</smiles>

Compound 3f :-

FTIR (KBr, $\left.\quad \mathbf{~ c m}^{-1}\right): \quad 1628(\mathrm{C}=\mathrm{O}) \quad, 1557(\mathrm{C}=\mathrm{C})$ ， 1425(C-C Aromatic str), 671(C-Br).

M.S. (m/z): 448(M-1).

${ }^{1}$ HNMR:- $\quad 2.45\left(\mathrm{~s}, \quad 3 \mathrm{H}, \quad \mathrm{CH}_{3}\right), \quad 7.46(\mathrm{~d}, \quad 1 \mathrm{H}$, $\mathrm{H \alpha}, \mathrm{J}=15 \mathrm{~Hz}), 7.48\left(\mathrm{~d}, 1 \mathrm{H}, \mathrm{H}_{2}\right), 7.56\left(\mathrm{~d}, 1 \mathrm{H}, \mathrm{H}_{1}\right), 7.87(\mathrm{~d}$, $1 \mathrm{H}, \mathrm{H} \beta, \mathrm{J}=15 \mathrm{~Hz}), 8.20\left(\mathrm{~s}, 1 \mathrm{H}, \mathrm{H}_{3}\right), 8.38\left(\mathrm{~s}, 1 \mathrm{H}, \mathrm{H}_{4}\right)$, 13.77(s, $1 \mathrm{H}, \mathrm{OH}$ ortho).

\section{Antimicrobial activity:}

Antimicrobial screening was done using disc diffusion method ${ }^{20}$ at a concentration of $100 \mu \mathrm{g} / \mathrm{ml}$.

Procedure:- The test was performed according to the disk diffusion method ${ }^{20}$ adopted with some modification for the prepared compound using Penciline and streptomycin as references. The prepared compounds were tested against one strain of Gram +ve bacteria, Gram -ve bactria, fungi. Whatman filter paper disk of $5 \mathrm{~mm}$ diameter were sterilized by autoclaving for $15 \mathrm{~min}$ at $121^{\circ} \mathrm{C}$. The sterile disk were impregnated with different compounds $(600 \mathrm{gm} / \mathrm{disk})$. Agar plates were surface inoculated uniformly from the both culture of the tested microorganism. The disk were placed on the medium suitably spaced apart on the plate were incubated at $50^{\circ} \mathrm{C}$ for $1 \mathrm{hr}$ to permit good diffusion and then transferred to an incubator at $37^{\circ} \mathrm{C}$. for $24 \mathrm{hr}$ for bacteria and $28^{\circ} \mathrm{C}$ for $72 \mathrm{hrs}$ for fungi.

The compounds were evaluated for antibacterial activity against Staphylococcus aureus gr +ve, Escherichia coli gr -ve Bacillus subtilis gr +ve, Salmonela typhi gr -ve, and antifungal activity against Aspergillus oryzoe, Aspergillus niger,. DMSO was used as solvent control. The results of antimicrobial data are summarized in table 2. The compounds show the moderate to good activity against bacteria and fungui. 
Table-2 Antimicrobial activity of synthesized Chalcones (3a-j).

\begin{tabular}{|c|c|c|c|c|c|c|}
\hline \multirow[t]{2}{*}{ compounds } & \multicolumn{2}{|c|}{$\begin{array}{c}\text { Gram positive } \\
\text { bacterias }\end{array}$} & \multicolumn{2}{|c|}{$\begin{array}{c}\text { Gram negative } \\
\text { bacterias }\end{array}$} & \multicolumn{2}{|c|}{ Fungus } \\
\hline & $\begin{array}{l}\text { Staph } \\
\text { aureus }\end{array}$ & $\begin{array}{l}\text { Bacillus } \\
\text { subtilis }\end{array}$ & $\begin{array}{l}\text { Escherichia } \\
\text { coli }\end{array}$ & $\begin{array}{c}\text { Pseudomonas } \\
\text { aeruginosa }\end{array}$ & $\begin{array}{l}\text { Aspergillus } \\
\text { oryzoe }\end{array}$ & $\begin{array}{l}\text { Aspergillus } \\
\text { niger, }\end{array}$ \\
\hline $3 a$ & + & + & - & - & - & - \\
\hline $3 \mathbf{b}$ & + & + & + & + & ++ & + \\
\hline $3 c$ & + & + & - & - & - & - \\
\hline $3 d$ & + & + & - & - & - & - \\
\hline $3 e$ & + & + & - & - & - & - \\
\hline $3 f$ & + & + & - & - & - & - \\
\hline $3 g$ & + & ++ & - & + & + & + \\
\hline $3 \mathrm{~h}$ & + & ++ & + & - & + & - \\
\hline $3 \mathbf{i}$ & + & + & - & + & + & + \\
\hline $3 \mathbf{j}$ & + & + & - & - & + & - \\
\hline Penciline 1 & + & + & + & + & $\mathrm{x}$ & $\mathbf{x}$ \\
\hline $\begin{array}{c}\text { Streptomycin } \\
2\end{array}$ & ++ & ++ & ++ & ++ & $\mathrm{x}$ & $\mathbf{x}$ \\
\hline Greseofulvin & $\mathrm{x}$ & $\mathrm{x}$ & $\mathrm{x}$ & $\mathrm{x}$ & - & - \\
\hline
\end{tabular}

\section{CONCLUSION:}

Successfully, in this work we have synthesized some novel chalcones using ortho hydroxy acetophenone with several aromatic aldehydes with high yield. The newly synthesized chalcones were confirmed by spectral analysis and further evaluated for their antimicrobial activity. The screening results revealed that the compounds $3 a-j$ showed significant antimicrobial activity.

\section{ACKNOWLEDGEMENT:}

The authors are thankful to Head of department of Chemistry, Head of department of Microbilogy, Principal of Yeshwant College, Nanded for providing lab facilities for the research work. 


\section{REFERENCES:}

1. Maria J.Gozalez Moa, Marcos Mandado et al. QTAIM electron density study of natural chalcones. Chemical physics letters, $2007,34,446$.

2. Awasthi SK, MishraN, KumarB, SharmaM, BhattacharyaA, MishraLC, BhasinVK, Potent antimalarial activity of newly synthesized substituted chalcone analogs in vitro. Medicinal Chemistry Research. 2009, 18:407-420.

3. Cheng. MS, ShiliR, KenyonG. A solid phase synthesis of chalcones by Claisen-Schmidt condensations. Chinese Chemical Letters, 2000, 11: 851-854.

4. LimSS, KimHS, LeeDU. In-vitro antimalarial activity of flavonoids and chalcones. Bulletin of theKorean Chemical Society, 2007, 28:2495-2497.

5. Straub, T. S. Tetrahedron Lett 1995, 36, 663.

6. Sandler, S., Karo, W. In Organic Functional Group Preparations. 1972, 3, 372.

7. Bergman, E. D., Ginsibm, L., Pappo, R. Org. React. 1959, 10, 179.

8. Ballesteros, J. F., Sanz, M.J., Ubeda, A., Miranda, M. A., Iborra, S., Paya, M., Alcaraz, M. J. Med. Chem. (1995), 38, 2794.

9. Go, M. L., Wu, X., Liu, X.L. Curr. Med. Chem. (2005), 12, 483.
10. Mukerjee, V. K., Prased, A. K., Raj, A. G., Brakhe, M. E., Olsen, C. E., Jain, S. C., Parmer, V. P. Bioorg. Med. Chem. (2001), 9,337 .

11. Liu, U. M., Wilairat, P., Croft, S. L., Tan, A. L., Go, M. Bioorg. Med .Chem. (2003),11, 2729.

12. Sivakumar, P. M., Geetha Babu, S. K., Mukesh, D. Chem. Pharm. Bull. (2007) ,55, 44.

13. Viana, G. S., Bandeira, M. A., Mantos, F. J. Phytomedicine. (2003), 10, 189.

14. Tiwari, N., Dwivedi, B., Nizamuddin, K. F., Nakanshi, Y., Lee, K. H. Bioorg .Med. Chem. (2000), 10, 699.

15. Ducki, S., Forrest, R., Hadfield, J. A., Kendall, A., Lawrence, N. J., Mc-Gown, A.T., Rennison, D. Bioorg. Med. Chem. (1998), 8, 1051.

16. H. Rupe and D. Wasserzug, J. Chem Ber., (1901), 34, 3527.

17. S. A. Hermes, Chem Ber., (1969), 70, 96422h.

18. D. S. Breslow and C. R. Houser, Chem Ber., (1940), 62, 2385.

19. K. Kazauki, K. Hitayama, S. Yokomor and T. Soki, Chem Abstr., (1976), 85, 591.

20. H.Afaf, El-masry, H.H. Fahmy and S.H. Ali, Abdelwahed, Molecules 2000, 5(12), 14291438 